\title{
Isolation, immunophenotypic characterization and pluripotency of dental pulp stem cells
}

\author{
Simone Bonato Luisi*1, Manoel Sant'Anna Filho' ${ }^{1}$ and Patricia Pranke ${ }^{2,3}$ \\ ${ }^{1}$ Department of Conservative Dentistry, Faculty of Dentistry, Universidade Federal do Rio Grande do Sul, Porto Alegre, Brazil \\ ${ }^{2}$ Head of Hematology and Stem Cell Laboratory, Faculty of Pharmacy, Universidade Federal do Rio Grande do Sul, Porto Alegre, Brazil \\ ${ }^{3}$ Stem Cell Research Institute, Porto Alegre, Brazil
}

\begin{abstract}
Objective: The aim of this study has been the isolation and characterization of stem cells from dental pulp (DPSCs) in culture.

Methods: The primary DPSCs cultures were obtained from human third molars. Immediately after extraction, the teeth were placed in Dulbecco's modified Eagle medium (DMEM) culture medium supplemented with fetal bovine serum and antibiotics. In a laminar flow, the pulp was removed from the tooth, incubated with collagenase for 2 hours and placed on a culture plate. Phenotypic characterization and cell pluripotency was performed in the fifth passage (P5). To evaluate the expression of surface markers, the cells were incubated with antibodies against CD14, CD29, CD34, CD44, CD45, CD73, CD90, CD105 and HLA-DR antigens. For induction of cell differentiation in vitro, $10^{4}$ cells $/ \mathrm{cm}^{2}$ were plated in 12 -well plates and cultivated in appropriate media for osteogenic, adipogenic and condrogencic differentiation after reaching at least $70 \%$ confluence.
\end{abstract}

Results: The cells were positive above 95\% for characteristic markers of the mesenchymal stem cells CD29, CD44, CD73 and CD90. In contrast, there was a low percentage of positivity (up to 1.1\%) for the characteristic markers of hematopoietic cells such as CD14, CD34, CD45, CD184 and HLA-DR. Adipogenic differentiation was visualized by staining lipid vacuoles with Oil Red. Bone differentiation was visualized by staining calcium deposits with Alizarin Red. No chondrogenic differentiation was observed.

Conclusions: The isolated cells were adherent to plastic, positive for the characteristic markers of mesenchymal stem cells and, therefore, an alternative source for tissue engineering studies.

\section{Introduction}

The pulp of human third molars is rich in mesenchymal stem cells, which is characterized by having high rates of proliferation and multipotentiality therefore having high potential for application in tissue engineering. The last permanent tooth to erupt is the third molar. This generally occurs between the age of 16 and 20. Third molars are often extracted for orthodontic reasons and discarded. They represent an important source of mesenchymal stem cells for research. Parameters have been used to characterize a population of mesenchymal stem cells. In 2006, the Mesenchymal and Tissue Stem Cell Committee of the International Society for Cellular Therapy established minimal criteria to define human MSCs, which include the following: a) capacity of adhesion to plastic when maintained in standard culture conditions; b) expression of CD105, CD73, and CD90 and lack of expression of CD45, CD34, CD14 or CD11b, CD79a or CD19, and HLA-DR surface molecules; c) differentiation into osteoblasts, adipocytes, and chondroblasts in vitro [1]. This study has aimed to evaluate the immunophenotypic profile and pluripotency of pulp cells from human third molars in culture.

\section{Materials and methods}

\section{Isolation and culture}

The cells were obtained from an impacted third molar extracted for orthodontic reasons. The donor (21 years old) was informed of the purposes of the study and signed an informed consent form. The research protocol was approved by the Research Ethics Committee at the Dentistry School - Universidade Federal do Rio Grande do Sul. A horizontal groove was gently cut on the root surface. The following procedures were performed in laminar flow under sterile conditions: the crown was separated from the root and the pulp was minced with a knife. The minced tissue was incubated at $37^{\circ} \mathrm{C}$ for 120 minutes in buffer containing $0.2 \%$ type 1 collagenase (Gibco, Grand Island, NY,USA). Cell suspension was centrifuged at $800 \mathrm{~g}$ for 10 minutes, at 4 ${ }^{\circ} \mathrm{C}$. The pellet was resuspended in Dulbecco's modified Eagle medium (DMEM, Gibco, Grand Island, NY,USA), supplemented with 10\% fetal calf serum, 100 units $/ \mathrm{mL}$ penicillin, $100 \mu \mathrm{g} / \mathrm{mL}$ streptomycin (Gibco, Grand Island, NY,USA), $0.45 \mu \mathrm{g} / \mathrm{mL}$ gentamicin (Shering-Plough, RJ, RJ, Brazil) and $3.7 \mathrm{mg} / \mathrm{L}$ HEPES (Gibco, Grand Island, NY,USA). The cells were cultivated six-well tissue culture plates (TPP Trasadingen, Schaffhausen, Switzerland) and incubated in $5 \% \mathrm{CO}_{2}$ at $37{ }^{\circ} \mathrm{C}$. The culture medium was changed every four days. When the cells became confluent, they were harvested with $0.5 \%$ trypsin-EDTA (SigmaAldrich/St Louis, Missouri, USA) and transferred to subcultures at a

Correspondence to: Simone Bonato Luisi, Department of Conservative Dentistry, Faculty of Dentistry, Universidade Federal do Rio Grande do Sul, Porto Alegre, BRAZIL, Rua Ramiro Barcelos, 2492, Porto Alegre, Rio Grande do Sul, Brazil, Tel: 90035003, E-mail: simone.luisi@ufrgs.br

Key words: cell culture, human dental pulp, stem cells

Received: April 21, 2017; Accepted: May 08, 2017; Published: May 11, 2017 
ratio of 1:3, in the same medium. The same procedure was followed each time confluence was reached.

\section{Cell differentiation in vitro}

To evaluate the ability of the cells to differentiate, $10^{4} \mathrm{cells} / \mathrm{cm}^{2}$ (at the fifth passage) were plated in 12-well plates and cultivated in appropriate media osteogenic, adipogenic and chondrogenic differentiation for 2 to 4 weeks after reaching at least $70 \%$ confluence [2]. For each experiment, a negative control was used consisting of the same cells maintained in conventional culture medium. After the period for differentiation, the cultures were washed with deionized water and fixed in $4 \%$ paraformaldehyde for for $20 \mathrm{~min}$ (osteogenic and chondrogenic) or $1 \mathrm{~h}$ (adipogenic). The cells that underwent osteogenic differentiation were stained with Alizarin Red, adipogenic differentiation with Oil Red, and chondrogenic differentiation with Alcian Blue.

\section{Flow cytometry}

For the evaluation of surface marker expression, 106 cells (at the fifth passage) were incubated with the following conjugated antibodies against human clusters of differentiation (CDs) and cell surface molecules: CD14/FITC, CD29/PE, CD34/PE, CD44/FITC, CD45/ FITC, CD73/PE, CD90/FITC, CD105 and human leukocyte antigen major hitocompatibility complex class II cell surface receptor (HLADR)/FITC (PharMingen-BD Bioscences, San Diego, California, USA) antigens. Unstained cells and cells labeled with mouse IgG1 isotype control conjugated with PE and FITC were used as the control. The 7-aminoactinomycin D (7AAD) is a nuclear stain for apoptosis, and used to separate dead cells from living cells. Data was acquired using the FACSAria flow cytometer (BD Bioscience, San Jose, California, USA) and 10,000 events were analyzed (FACSDiva 6.1.3-BD Bioscience, San Jose, California, USA).

\section{Results}

The isolated cells in primary culture were adherent to plastic and had fibroblast-like morphology. The cells (passage 5 - P5) were positive above $95 \%$ for characteristic markers of mesenchymal stem cells CD29, CD44, CD73 and CD90. In contrast, a low percentage of positivity was detected (1.1\%) for the characteristic markers of hematopoietic cells such as CD14, CD34, CD45, CD184 and HLA-DR (Figure 1).
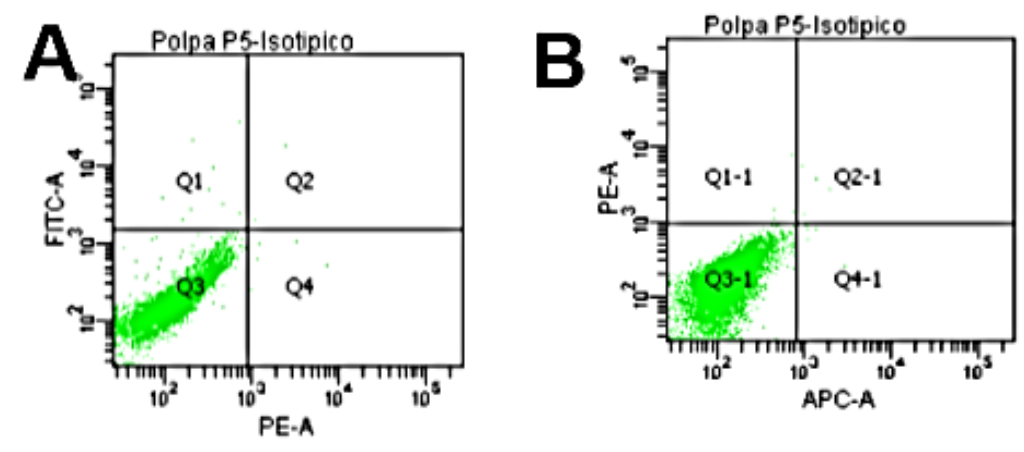

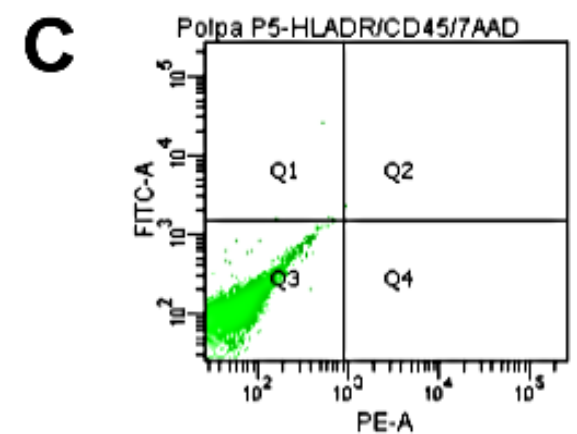

PE-A
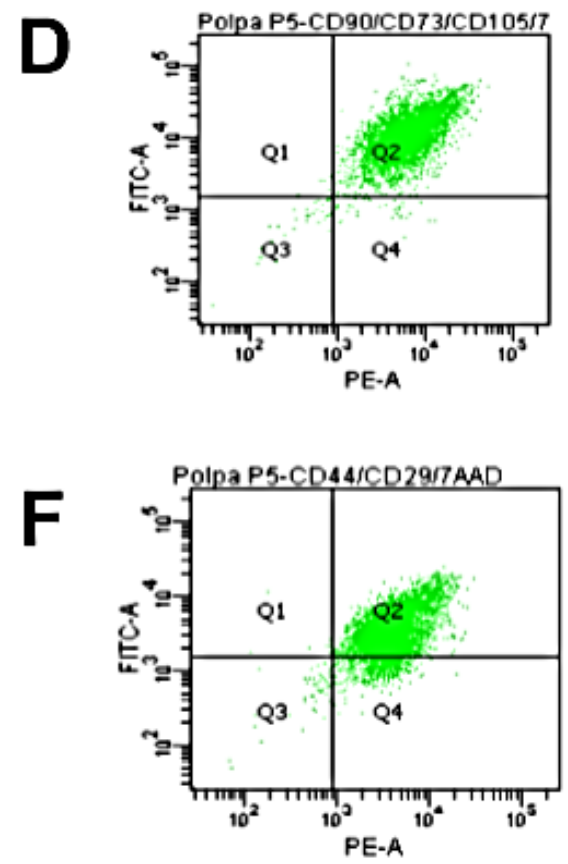
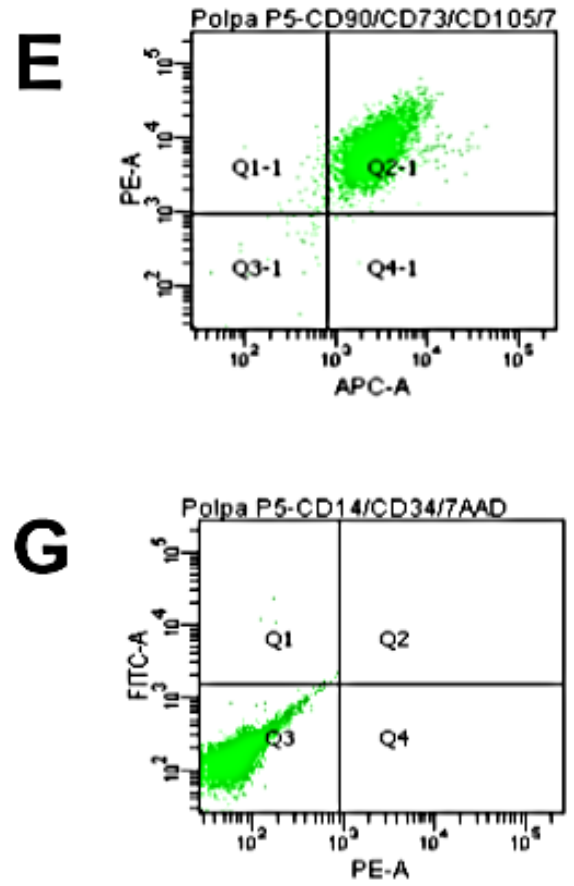

Figure 1. Analysis of surface markers DPSC in the fifth passage by flow cytometry. Diagrams show isotype antibodies (A, B), HLA-DR and CD45 (C), CD90 and CD73 (D), CD73 and CD105 (E), CD44 and CD29 (F) CD14 and CD34 (G). 
Adipogenic differentiation was visualized by staining lipid vacuoles with Oil Red. Bone differentiation was visualized by staining calcium deposits with Alizarin Red. It was not possible to verify chondrogenic differentiation.

\section{Discussion}

The first type of dental stem cell was isolated from normal human impacted third molars in 2000. The dental pulp stem cells (DPSCs) were isolated from the pulp tissue of a human impacted third molar by enzymatic treatment [3]. Third molars are often extracted for orthodontic reasons and discarded. These theeth represent an important source of mesenchymal stem cells for studies in tissue engineering and regenerative medicine as they have the advantage of being a non-invasive and disposable source of cells. Many studies have demonstrated that DPSCs have the ability to perform self renewal and differentiate into osteogenic, dentinogenic, neurogenic, and myogenic cell lineages in different inductive media [4-8].

Studies have shown that extracted impacted third molars are capable of producing an optimum quantity of dental pulp tissue for the isolation of DPSCs $[9,10]$. Various methods have been described for isolation of stem cells from human dental pulp. Raoof, et al. [11]. used three different methods for isolation of DPSCs from dental pulp tissue: 1 . Dental pulp tissue was digested with collagenase or dispase enzyme and isolated trypsinised cells were plated in culture dishes; 2. Small undigested dental pulp tissue pieces were explanted directly to petri dishes; and 3. Dental pulp tissue was initially trypsinised and then small tissue pieces explanted to petri dishes for their outgrowth. The authors have recommended the third method for the isolation of dental pulp DPSC because it demonstrated high efficiency in a short time. Huang, et al. [12]. compared the enzyme digestion and outgrowth methods and found that cells isolated by enzyme digestion had a higher proliferation rate than those isolated by the other methods. In this study, the enzymatic digestion method used in the studies of $Y u$, et al. [13], Bernardi, et al. [14], Werle, et al. [2], and their colleagues, was employed.

DPSCs, a type of adult stem cells, usually perform asymmetric cell division, which gives rise to one daughter cell with a stem-cell fate and another which can pursue further cell divisions to generate differentiated progenies. DPSCs in vivo usually remain quiescent within adult dental pulps, but respond during injury to produce progenies with high proliferative potential, which can differentiate into terminally differentiated odontoblasts. Thus, the amount of DPSCs in the normal dental pulp remains relatively constant [13].

The isolated dental pulp cells expressed mesenchymal stem cell markers. The cells were positive above $95 \%$ for characteristic markers of mesenchymal stem cells CD29, CD44, CD73 and CD90, which corroborate with the studies of Gronthos [3], Agha-Hosseini, et al. [15], and Chen, et al. [16].

In the present study, it was not possible to verify chondrogenic differentiation. Azin Malekfar, et al. [17] with the aim of isolating and characterizing human dental pulp stem cells from cryopreserved pulp tissue obtained from teeth with irreversible pulpitis, induced adipogenic and osteogenic differentiation and did not test the chondrogenic lineage.

In this study, isolated cells with fibroblast-like morphology had the ability to adhere to plastic when cultured and were positive for markers characteristic of mesenchymal stem cells. Although the pulp of human third molars has a low number of cells, the strong ability of these cells to proliferate makes them an alternative source for tissue engineering studies.

\section{Conflicts of interest}

The authors declare no conflicts of interest related to this article.

\section{References}

1. Dominici M, Le Blanc K, Mueller I, Slaper-Cortenbach I, Marini F, et al. (2006) Minimal criteria for defining multipotent mesenchymal stromal cells. The International Society for Cellular Therapy position statement. Cytotherapy 8: 315-317. [Crossref]

2. Werle SB, Lindemann D, Steffens D, Demarco FF, Araujo FB, et al. (2016) Carious deciduous teeth are a potential source for dental pulp stem cells. Clin Oral Invest 20: 75-81. [Crossref]

3. Gronthos S, Mankani M, Brahim J, Robey PG, Shi S (2000) Postnatal human dental pulp stem cells (DPSCs) in vitro and in vivo. Proc Natl Acad Sci U S A 97: 1362513630. [Crossref]

4. Gronthos S1, Brahim J, Li W, Fisher LW, Cherman N, et al. (2002) Stem cell properties of human dental pulp stem cells. J Dent Res 81: 531-535. [Crossref]

5. Iohara K, Zheng L, Ito M, Tomokiyo A, Matsushita K, et al. (2006) Side population cells isolated from porcine dental pulp tissue with self-renewal and multipotency for dentinogenesis, chondrogenesis, adipogenesis, and neurogenesis. Stem Cells 24: 2493 2503. [Crossref]

6. Jo YY, Lee HJ, Kook SY, Choung HW, Park JY, et al. (2007) Isolation and characterization of postnatal stem cells from human dental tissues. Tissue Eng 13: 767773. [Crossref]

7. Arthur A, Rychkov G, Shi S, Koblar SA, Gronthos S (2008) Adult human dental pulp stem cells differentiate toward functionally active neurons under appropriate environmental cues. Stem Cells 26: 1787-1795. [Crossref]

8. Huang GT, Gronthos S, Shi S (2009) Mesenchymal stem cells derived from denta tissues vs. those from other sources: their biology and role in regenerative medicine. $J$ Dent Res 88: 792-806. [Crossref]

9. Yalvac ME, Ramazanoglu M, Rizvanov AA, Sahin F, Bayrak OF, et al. (2010) Isolation and characterization of stem cells derived from human third molar tooth germs of young adults: implications in neo-vascularization, osteo-, adipo- and neurogenesis. Pharmacogenomics $J$ 10: 105-113. [Crossref]

10. Atari M, Barajas M, Hernández-Alfaro F, Gil C, Fabregat M, et al. (2011) Isolation of pluripotent stem cells from human third molar dental pulp. Histol Histopathol 26: 1057-1070. [Crossref]

11. Raoof M, Yaghoobi MM, Derakhshani A, Kamal-Abadi AM, Ebrahimi B, et al. (2014) A modified efficient method for dental pulp stem cell isolation. Dent Res $J$ (Isfahan) 11: 244-250. [Crossref]

12. Huang GT, Sonoyama W, Chen J, Park SH (2006) In vitro characterization of human dental pulp cells: various isolation methods and culturing environments. Cell Tissue Res 324: 225-236. [Crossref]

13. Yu J, He H, Tang C, Zhang G, Li Y, et al. (2010) Differentiation potential of STRO-1+ dental pulp stem cells changes during cell passaging. BMC Cell Biol 11: 32. [Crossref]

14. Bernardi L, Luisi SB, Fernandes R, Dalberto TP, Valentim L, et al. (2011) The isolation of stem cells from human deciduous teeth pulp is related to the physiological process of resorption. J Endod 37: 973-979. [Crossref]

15. Agha-Hosseini F, Jahani MA, Jahani M, Mirzaii-Dizgah I, Ali-Moghaddam K (2010) In vitro isolation of stem cells derived from human dental pulp. Clin Transplant 24: E23-28. [Crossref]

16. Chen G, Sun Q, Xie L, Jiang Z, Feng L, et al. (2015) Comparison of the odontogenic differentiation potential of dental follicle, dental papilla, and cranial neural crest cells. J Endod 41: 1091-1099. [Crossref]

17. Malekfar A, Valli KS, Kanafi MM, Bhonde RR (2016) Isolation and characterization of human dental pulp stem cells from cryopreserved pulp tissues obtained from teeth with irreversible pulpitis. J Endod 42: 76-81. [Crossref]

Copyright: (C2017 Luisi SB. This is an open-access article distributed under the terms of the Creative Commons Attribution License, which permits unrestricted use, distribution, and reproduction in any medium, provided the original author and source are credited. 